\title{
The relativistic inverse scattering problem for quantum graphs
}

\author{
K. K. Sabirov ${ }^{1}$, Z. A. Sobirov ${ }^{1}$, O. V. Karpova ${ }^{1,2}$, A. A. Saidov ${ }^{2}$ \\ ${ }^{1}$ National University of Uzbekistan, 4 University St., 100074, Tashkent, Uzbekistan \\ ${ }^{2}$ Turin Polytechnic University in Tashkent, 17 Niyazov St., 100095, Tashkent, Uzbekistan \\ karimjonsabirov@yahoo.com
}

PACS 02.30.Zz, 03.65.Pm

DOI 10.17586/2220-8054-2015-6-2-192-197

In this paper, we treat the inverse scattering problem for the Dirac equation on metric graphs. Using the known scattering data, we recover the potential in the Dirac equation. The Gel'fand-Levitan-Marchenko integral equation is derived and potential is explicitly obtained for the case of a primary star graph.

Keywords: quantum graph, inverse scattering problem, Dirac equation.

Received: 2 February 2015

\section{Introduction}

The study of particle and wave transport in branched structures and networks is of fundamental and practical importance in different topics of contemporary physics. In particular, transport in such nanoscale systems as quantum networks, molecular chains, networks of carbon nanotubes or quantum wires and the problem of charge transport in a DNA double helix can be successfully modeled by quantum mechanical wave equations on metric graphs. Initially, the concept of quantum graphs was introduced by Pauling more than half a century ago to describe electron transport in complex molecular chains of organic molecules. The difference between the usual graph and the quantum graph (both from the mathematical term) is that the dynamics of particles in such systems are described with quantum-mechanical wave equations. Quantum graphs can be considered as a class of confined quantum systems where the dynamics strongly depends on the topology of a graph. The topology of the graph can be given in terms of adjacency matrix:

$$
C_{i, j}=C_{j, i}= \begin{cases}1, & \text { if } i, j \text { are connected, } \\ 0, & \text { otherwise }\end{cases}
$$

Direct spectral and scattering problems on quantum graphs have been studied earlier in the context of quantum chaos theory (see reviews [1,2] and references therein). The inverse problem for quantum graphs can be divided into two types: the problem of the recovering the potential in the wave equation and the problem of finding the topology of a graph using the given spectral data. The latter is a more difficult problem because of possible isospectrality of graphs for different topologies [3]. In this paper, we solve the inverse scattering problem for the massless Dirac equation, given on the graph of the simplest topology, the so-called star graph. The importance of this problem is a result of its application for modeling charge, information and spin transport in branched nanostrctures (e.g., networks of graphene nanoribbons, carbon nanotubes (CNT) and quantum wires). The dynamics of quasi-particles in such systems are described by the massless Dirac equation. In this paper, we propose an 
The relativistic inverse scattering problem for quantum graphs

algorithm for reconstructing the potential of the Dirac equation using the available elements of the scattering matrix.

\section{Formulation of the problem}

Consider a star graph consisting of three semi-infinite edges connected at one point (see. Fig. 1). On each edge, we $b_{j}$ determine the coordinates $x_{j} \in[0, \infty)$ where $x_{j}=0$ corresponds to the vertex of the graph. The Dirac equation on the each edge $b_{j}$ is given as:

$$
\left(\begin{array}{cc}
-1 & 0 \\
0 & 1
\end{array}\right) \frac{d}{d x} f_{j}\left(x_{j}\right)+\left(\begin{array}{cc}
0 & q_{j}\left(x_{j}\right) \\
-p_{j}\left(x_{j}\right) & 0
\end{array}\right) f_{j}\left(x_{j}\right)=i \zeta f_{j}\left(x_{j}\right)
$$

where $f_{j}\left(x_{j}\right)=\left(\begin{array}{c}f_{j, 1}\left(x_{j}\right) \\ f_{j, 2}\left(x_{j}\right)\end{array}\right), j=1,2,3$. The following boundary conditions are imposed at the branching point:

$$
\begin{aligned}
& f_{1,1}(0)=f_{2,1}(0)=f_{3,1}(0), \\
& f_{1,2}(0)+f_{2,2}(0)+f_{3,2}(0)=0 .
\end{aligned}
$$

Let us define scattering matrices on each bond as:

$$
S_{j}\left(\zeta_{j}\right)=\left(\begin{array}{cc}
a_{j}(\zeta) & b_{j}(\zeta) \\
\bar{b}_{j}(\zeta) & -\bar{a}_{j}(\zeta)
\end{array}\right), j=1,2,3
$$

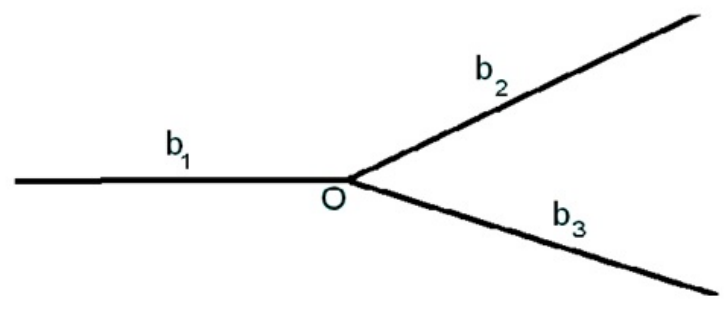

FIG. 1. Star graph

\section{The direct scattering problem}

We find three solutions $f^{(m)}(x, \zeta)=\left\{f_{1}^{(m)}\left(x_{1}, \zeta\right), f_{2}^{(m)}\left(x_{2}, \zeta\right), f_{3}^{(m)}\left(x_{3}, \zeta\right)\right\}, m=1,2,3$ for the problem given by Eqs. (1)-(3). Furthermore, we define the functions $\psi_{j}\left(x_{j}, \zeta\right), \bar{\psi}_{j}\left(x_{j}, \zeta\right)$ as solutions of the following integral equations:

$$
\begin{aligned}
\psi_{j, 1}\left(x_{j}, \zeta\right) & =\int_{x_{j}}^{+\infty} q_{j}(t) e^{-i \zeta\left(x_{j}-t\right)} \psi_{j, 2}(t, \zeta) d t \\
\psi_{j, 2}\left(x_{j}, \zeta\right) & =e^{i \zeta x_{j}}+\int_{x_{j}}^{+\infty} \int_{t}^{+\infty} p_{j}(t) q_{j}(z) e^{-i \zeta\left(2 t-x_{j}-z\right)} \psi_{j, 2}(z, \zeta) d z d t, \\
\bar{\psi}_{j, 1}\left(x_{j}, \zeta\right) & =e^{-i \zeta x_{j}}+\int_{x_{j}}^{+\infty} \int_{t}^{+\infty} q_{j}(t) p_{j}(z) e^{i \zeta\left(2 t-x_{j}-z\right)} \bar{\psi}_{j, 1}(z, \zeta) d z d t \\
\bar{\psi}_{j, 2}\left(x_{j}, \zeta\right) & =\int_{x_{j}}^{+\infty} p_{j}(t) e^{i \zeta\left(x_{j}-t\right)} \bar{\psi}_{j, 1}(t, \zeta) d t .
\end{aligned}
$$

Also, we introduce the solutions $\varphi_{j}\left(x_{j}, \zeta\right), \bar{\varphi}_{j}\left(x_{j}, \zeta\right)$ obeying the following integral equations:

$$
\begin{aligned}
\varphi_{j, 1}\left(x_{j}, \zeta\right) & =e^{-i \zeta x_{j}}+\int_{0}^{x_{j}} \int_{0}^{t} q_{j}(t) p_{j}(z) e^{i \zeta\left(2 t-x_{j}-z\right)} \varphi_{j, 1}(z, \zeta) d z d t \\
\varphi_{j, 2}\left(x_{j}, \zeta\right) & =\int_{0}^{x} p_{j}(t) e^{i \zeta\left(x_{j}-t\right)} \varphi_{j, 1}(t, \zeta) d t
\end{aligned}
$$




$$
\begin{aligned}
& \bar{\varphi}_{j, 1}\left(x_{j}, \zeta\right)=\int_{0}^{x_{j}} q_{j}(t) e^{-i \zeta\left(x_{j}-t\right)} \bar{\varphi}_{j, 2}(t, \zeta) d t \\
& \bar{\varphi}_{j, 2}\left(x_{j}, \zeta\right)=-e^{i \zeta x_{j}}+\int_{0}^{x} \int_{0}^{t} p_{j}(t) q_{j}(z) e^{-i \zeta\left(2 t-x_{j}-z\right)} \bar{\varphi}_{j, 2}(z, \zeta) d z d t .
\end{aligned}
$$

It is easy to see that if $q_{j}, p_{j} \in L_{1}$, then the functions $e^{i \zeta x_{j}} \varphi_{j}\left(x_{j}, \zeta\right), e^{-i \zeta x_{j}} \psi_{j}\left(x_{j}, \zeta\right)$ are analytic on the upper plane $\operatorname{Im} \zeta>0, e^{-i \zeta x_{j}} \bar{\varphi}_{j}\left(x_{j}, \zeta\right), \quad e^{i \zeta x_{j}} \bar{\psi}_{j}\left(x_{j}, \zeta\right)$ are analytic on the lower plane $\operatorname{Im} \zeta<0$.

Since the functions $\psi_{j}\left(x_{j}, \zeta\right), \bar{\psi}_{j}\left(x_{j}, \zeta\right)$ obey the boundary conditions:

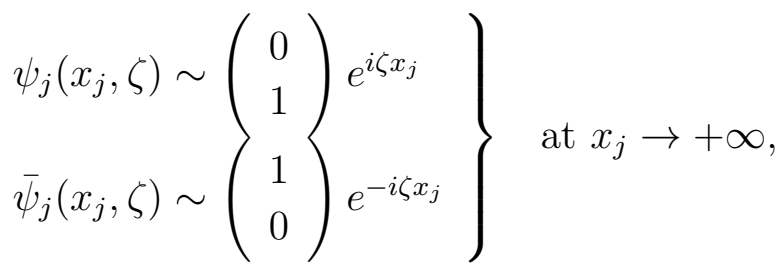

the solutions $\psi_{j}\left(x_{j}, \zeta\right), \bar{\psi}_{j}\left(x_{j}, \zeta\right)$ are linearly independent. This follows from the condition for the Wronskian: $W\left(\psi_{j}, \bar{\psi}_{j}\right)=-1$. Therefore we have:

$$
\begin{aligned}
& \varphi_{j}=a_{j}(\zeta) \bar{\psi}_{j}+b_{j}(\zeta) \psi_{j}, \\
& \bar{\varphi}_{j}=-\bar{a}_{j}(\zeta) \psi_{j}+\bar{b}_{j}(\zeta) \bar{\psi}_{j} .
\end{aligned}
$$

It follows from Eqs. (6)-(7) that functions $\varphi_{j}\left(x_{j}, \zeta\right), \bar{\varphi}_{j}\left(x_{j}, \zeta\right)$ satisfy conditions:

$$
\begin{aligned}
& \varphi_{j}(0, \zeta)=\left(\begin{array}{c}
1 \\
0
\end{array}\right), \\
& \bar{\varphi}_{j}(0, \zeta)=\left(\begin{array}{c}
0 \\
-1
\end{array}\right) .
\end{aligned}
$$

Furthermore, from $W\left(\varphi_{j}, \bar{\varphi}_{j}\right)=-1$ we have:

$$
a_{j} \bar{a}_{j}+b_{j} \bar{b}_{j}=1
$$

which implies that:

$$
\begin{aligned}
\psi_{j}(0, \zeta) & =\left(\begin{array}{c}
\bar{b}_{j} \\
a_{j}
\end{array}\right), \\
\bar{\psi}_{j}(0, \zeta) & =\left(\begin{array}{c}
\bar{a}_{j} \\
-b_{j}
\end{array}\right) .
\end{aligned}
$$

The solutions $f^{(m)}(x, \zeta)=\left\{f_{1}^{(m)}\left(x_{1}, \zeta\right), f_{2}^{(m)}\left(x_{2}, \zeta\right), f_{3}^{(m)}\left(x_{3}, \zeta\right)\right\}, m=1,2,3$ can be written as:

$$
\begin{aligned}
& f_{j}^{(m)}\left(x_{j}, \zeta\right)=t_{j}^{(m)}(\zeta) \bar{\psi}_{j}\left(x_{j}, \zeta\right), m \neq j, j=1,2,3, \\
& f_{m}^{(m)}\left(x_{m}, \zeta\right)=\varphi_{m}\left(x_{m}, \zeta\right)+r_{m}^{(m)}(\zeta) \psi_{m}\left(x_{m}, \zeta\right) e^{-2 i \zeta x_{m}}, m=1,2,3 .
\end{aligned}
$$

The coefficients $r_{m}^{(m)}, t_{j}^{(m)}(m \neq j, \quad m, j=1,2,3)$ can be found from the boundary conditions for $f^{(m)}(x, \zeta)$. For example, at $m=1$ we have:

$$
\begin{aligned}
& \varphi_{1,1}(0, \zeta)+r_{1}^{(1)}(\zeta) \psi_{1,1}(0, \zeta)=t_{2}^{(1)}(\zeta) \bar{\psi}_{2,1}(0, \zeta)=t_{3}^{(1)}(\zeta) \bar{\psi}_{3,1}(0, \zeta), \\
& \varphi_{1,2}(0, \zeta)+r_{1}^{(1)}(\zeta) \psi_{1,2}(0, \zeta)+t_{2}^{(1)}(\zeta) \bar{\psi}_{2,2}(0, \zeta)+t_{3}^{(1)}(\zeta) \bar{\psi}_{3,2}(0, \zeta)=0 .
\end{aligned}
$$


Using Eqs. (11)-(12), one can obtain:

$$
\begin{aligned}
& r_{1}^{(1)}(\zeta)=\frac{\bar{a}_{2} b_{3}+b_{2} \bar{a}_{3}}{a_{1} \bar{a}_{2} \bar{a}_{3}-\bar{b}_{1} \bar{a}_{2} b_{3}-\bar{b}_{1} b_{2} \bar{a}_{3}}, \\
& t_{2}^{(1)}(\zeta)=\frac{a_{1} \bar{a}_{3}}{a_{1} \bar{a}_{2} \bar{a}_{3}-\bar{b}_{1} \bar{a}_{2} b_{3}-\bar{b}_{1} b_{2} \bar{a}_{3}}, \\
& t_{3}^{(1)}(\zeta)=\frac{a_{1} \bar{a}_{2}}{a_{1} \bar{a}_{2} \bar{a}_{3}-\bar{b}_{1} \bar{a}_{2} b_{3}-\bar{b}_{1} b_{2} \bar{a}_{3}} .
\end{aligned}
$$

The above analysis is usually called the direct scattering problem. In the following, we will treat the inverse scattering problem.

\section{The inverse scattering problem}

Using the following integral representations for the functions $\psi_{j}\left(x_{j}, \zeta\right), \bar{\psi}_{j}\left(x_{j}, \zeta\right)$ :

$$
\begin{gathered}
\psi_{j}(x, \zeta)=\left(\begin{array}{l}
0 \\
1
\end{array}\right) e^{i \zeta x_{j}}+\int_{x_{j}}^{+\infty} K_{j}\left(x_{j}, s\right) e^{i \zeta s} d s, \\
\bar{\psi}_{j}(x, \zeta)=\left(\begin{array}{l}
1 \\
0
\end{array}\right) e^{-i \zeta x_{j}}+\int_{x_{j}}^{+\infty} \bar{K}_{j}\left(x_{j}, s\right) e^{-i \zeta s} d s,
\end{gathered}
$$

where $K_{j}\left(x_{j}, s\right)=\left(\begin{array}{c}K_{j, 1}\left(x_{j}, s\right) \\ K_{j, 2}\left(x_{j}, s\right)\end{array}\right), \bar{K}_{j}\left(x_{j}, s\right)=\left(\begin{array}{c}\bar{K}_{j, 1}\left(x_{j}, s\right) \\ \bar{K}_{j, 2}\left(x_{j}, s\right)\end{array}\right)$ are two-component vectors. To obey fy the boundary conditions (8), we assume that $K_{j}\left(x_{j}, s\right)=0$ at $x_{j}>s$. Now, we will prove the existence of representations (16)-(17). To do that, it is enough to insert Eqs. (16)-(17) into (1). Then, from Eq. (16) we obtain:

$$
\begin{aligned}
& \int_{x_{j}}^{+\infty} e^{i \zeta s}\left[\left(\partial_{x_{j}}-\partial_{s}\right) K_{j, 1}\left(x_{j}, s\right)-q_{j}\left(x_{j}\right) K_{j, 2}\left(x_{j}, s\right)\right] d s- \\
& -\left[q_{j}\left(x_{j}\right)+2 K_{j, 1}\left(x_{j}, x_{j}\right)\right] e^{i \zeta x_{j}}+\lim _{s \rightarrow \infty}\left[K_{j, 1}\left(x_{j}, s\right) e^{i \zeta s}\right]=0 \\
& \int_{x_{j}}^{+\infty} e^{i \zeta s}\left[\left(\partial_{x_{j}}+\partial_{s}\right) K_{j, 2}\left(x_{j}, s\right)-p_{j}\left(x_{j}\right) K_{j, 1}\left(x_{j}, s\right)\right] d s- \\
& -\lim _{s \rightarrow \infty}\left[K_{j, 2}\left(x_{j}, s\right) e^{i \zeta s}\right]=0
\end{aligned}
$$

Thus, for the existence of representations (16)-(17), it is necessary and sufficient that functions $K_{j}\left(x_{j}, s\right)$ obey the following equations:

$$
\begin{aligned}
& \left(\partial_{x_{j}}-\partial_{s}\right) K_{j, 1}\left(x_{j}, s\right)-q_{j}\left(x_{j}\right) K_{j, 2}\left(x_{j}, s\right)=0, \\
& \left(\partial_{x_{j}}+\partial_{s}\right) K_{j, 2}\left(x_{j}, s\right)-p_{j}\left(x_{j}\right) K_{j, 1}\left(x_{j}, s\right)=0,
\end{aligned}
$$

with the boundary conditions:

$$
\begin{aligned}
& K_{j, 1}\left(x_{j}, x_{j}\right)=-\frac{1}{2} q_{j}\left(x_{j}\right), \\
& \lim _{s \rightarrow+\infty} K_{j}\left(x_{j}, s\right)=0 .
\end{aligned}
$$

Solutions for problems (20)-(21) (the Gursah problem) exist and are unique. The above treatment can be done for the function $\bar{K}_{j}\left(x_{j}, s\right)$, as well.

Let given $C_{j}$ contour that begins from $-\infty+i 0^{+}$, rounds above all zeros of $a_{j}(\zeta)$ and poles of $r_{j}^{(j)}(\zeta)$, and ends at $+\infty+i 0^{+}$. We introduce the following function:

$$
g_{1}^{(1)}\left(x_{1}, \zeta\right)=\varphi_{1}\left(x_{1}, \zeta\right)+r_{1}^{(1)}(\zeta) \psi_{1}\left(x_{1}, \zeta\right) e^{-2 i \zeta x_{1}}
$$

Then, from Eq. (9) we have:

$$
\begin{aligned}
& g_{1}^{(1)}\left(x_{1}, \zeta\right)=\varphi_{1}\left(x_{1}, \zeta\right)+r_{1}^{(1)}(\zeta) \psi_{1}\left(x_{1}, \zeta\right) e^{-2 i \zeta x_{1}}= \\
& =a_{1}(\zeta) \bar{\psi}_{1}\left(x_{1}, \zeta\right)+\left\{b_{1}(\zeta)+r_{1}^{(1)}(\zeta) e^{-2 i \zeta x_{1}}\right\} \psi_{1}\left(x_{1}, \zeta\right)
\end{aligned} .
$$


The last equation can be rewritten as:

$$
\begin{aligned}
\frac{g_{1}^{(1)}\left(x_{1}, \zeta\right)}{a_{1}(\zeta)}= & \bar{\psi}_{1}\left(x_{1}, \zeta\right)+\left\{\frac{b_{1}(\zeta)}{a_{1}(\zeta)}+\frac{r_{1}^{(1)}(\zeta)}{a_{1}(\zeta)} e^{-2 i \zeta x_{1}}\right\} \psi_{1}\left(x_{1}, \zeta\right)= \\
= & \bar{\psi}_{1}\left(x_{1}, \zeta\right)+R_{1}^{(1)}\left(x_{1}, \zeta\right) \psi_{1}\left(x_{1}, \zeta\right)
\end{aligned}
$$

where $R_{1}^{(1)}\left(x_{1}, \zeta\right)=\frac{b_{1}(\zeta)}{a_{1}(\zeta)}+\frac{r_{1}^{(1)}(\zeta)}{a_{1}(\zeta)} e^{-2 i \zeta x_{1}}$. Inserting Eqs. (16) and (17) into (24):

$$
\begin{aligned}
& \frac{g_{1}^{(1)}\left(x_{1}, \zeta\right)}{a_{1}(\zeta)}=\left(\begin{array}{l}
1 \\
0
\end{array}\right) e^{-i \zeta x_{1}}+\int_{x_{1}}^{+\infty} \bar{K}_{1}\left(x_{1}, s\right) e^{-i \zeta s} d s+ \\
& \quad+R_{1}^{(1)}\left(x_{1}, \zeta\right)\left(\left(\begin{array}{c}
0 \\
1
\end{array}\right) e^{i \zeta x_{1}}+\int_{x_{1}}^{+\infty} K_{1}\left(x_{1}, s\right) e^{i \zeta s} d s\right)
\end{aligned}
$$

multiplying Eq. (25) by $\frac{1}{2 \pi} e^{i \zeta y} d \zeta$ and integrating along the contour $C_{1}$ at $y>x_{1}$, by taking into account the representation $\delta(x)=\frac{1}{2 \pi} \int_{C_{1}} e^{i \zeta x} d \zeta$ the Dirac- $\delta$ function we have:

$$
\begin{aligned}
& \frac{1}{2 \pi} \int_{C_{1}} \frac{g_{1}^{(1)}\left(x_{1}, \zeta\right)}{a_{1}(\zeta)} e^{i \zeta y} d \zeta=\left(\begin{array}{c}
1 \\
0
\end{array}\right) \delta\left(y-x_{1}\right)+\int_{x_{1}}^{+\infty} \bar{K}_{1}\left(x_{1}, s\right) \delta(y-s) d s+ \\
& +\left(\begin{array}{c}
0 \\
1
\end{array}\right)\left[\frac{1}{2 \pi} \int_{C_{1}} R_{1}^{(1)}\left(x_{1}, \zeta\right) e^{i \zeta\left(x_{1}+y\right)} d \zeta\right]+\int_{x_{1}}^{+\infty} K_{1}\left(x_{1}, s\right)\left[\frac{1}{2 \pi} \int_{C_{1}} R_{1}^{(1)}\left(x_{1}, \zeta\right) e^{i \zeta(y+s)} d \zeta\right] d s
\end{aligned}
$$

Since the functions $e^{i \zeta x_{j}} \varphi_{j}\left(x_{j}, \zeta\right), \quad e^{-i \zeta x_{j}} \psi_{j}\left(x_{j}, \zeta\right)$ are analytic on the upper half of the plane $\operatorname{Im} \zeta>0, y>x_{1}$ and the contour $C_{1}$ round above zeros of $a_{1}(\zeta)$ and poles of $r_{1}^{(1)}(\zeta)$, the integral of the left hand side of equality (26) is equal to zero. Therefore we get:

$$
\bar{K}_{1}\left(x_{1}, y\right)+\left(\begin{array}{l}
0 \\
1
\end{array}\right) F_{1}\left(x_{1}, x_{1}+y\right)+\int_{x_{1}}^{+\infty} K_{1}\left(x_{1}, s\right) F_{1}\left(x_{1}, y+s\right) d s=0,
$$

where $F_{1}(x, y)=\frac{1}{2 \pi} \int_{C_{1}} R_{1}^{(1)}(x, \zeta) e^{i \zeta y} d \zeta$.

Repeating the same procedure for the function $\bar{f}_{1}^{(1)}(x, \zeta)$, we obtain:

$$
K_{1}\left(x_{1}, y\right)-\left(\begin{array}{l}
0 \\
1
\end{array}\right) \bar{F}_{1}\left(x_{1}, x_{1}+y\right)-\int_{x_{1}}^{+\infty} \bar{K}_{1}\left(x_{1}, s\right) \bar{F}_{1}\left(x_{1}, y+s\right) d s=0,
$$

where $\bar{F}_{1}(x, y)=\frac{1}{2 \pi} \int_{\bar{C}_{1}} \bar{R}_{1}^{(1)}(x, \zeta) e^{-i \zeta y} d \zeta$ and $\bar{C}_{1}$ is the same contour as $C_{1}$, but rounds below the zeros of $\bar{a}_{1}(\zeta)$ and poles of $\bar{r}_{1}^{(1)}(\zeta)$.

Similarly, one can obtain the following equations for the functions $K_{2}$ and $K_{3}$ :

$$
\begin{aligned}
& \bar{K}_{j}\left(x_{j}, y\right)+\left(\begin{array}{l}
0 \\
1
\end{array}\right) F_{j}\left(x_{j}, x_{j}+y\right)+\int_{x_{j}}^{+\infty} K_{j}\left(x_{j}, s\right) F_{j}\left(x_{j}, y+s\right) d s=0 \\
& K_{j}\left(x_{j}, y\right)-\left(\begin{array}{l}
0 \\
1
\end{array}\right) \bar{F}_{j}\left(x_{j}, x_{j}+y\right)-\int_{x_{j}}^{+\infty} \bar{K}_{j}\left(x_{j}, s\right) \bar{F}_{j}\left(x_{j}, y+s\right) d s=0
\end{aligned}
$$

where

$$
\begin{gathered}
F_{j}(x, y)=\frac{1}{2 \pi} \int_{C_{j}} R_{j}^{(j)}(x, \zeta) e^{i \zeta y} d \zeta, \bar{F}_{j}(x, y)=\frac{1}{2 \pi} \int_{\bar{C}_{j}} \bar{R}_{j}^{(j)}(x, \zeta) e^{-i \zeta y} d \zeta \\
R_{j}^{(j)}(x, \zeta)=\frac{b_{j}(\zeta)}{a_{j}(\zeta)}+\frac{r_{j}^{(j)}(\zeta)}{a_{j}(\zeta)} e^{-2 i \zeta x_{j}}, \bar{R}_{j}^{(j)}(x, \zeta)=\frac{\bar{b}_{j}(\zeta)}{\bar{a}_{j}(\zeta)}+\frac{\bar{r}_{j}^{(j)}(\zeta)}{\bar{a}_{j}(\zeta)} e^{2 i \zeta x_{j}}, j=2,3 .
\end{gathered}
$$


From Eq. (21), we obtain:

$$
q_{j}\left(x_{j}\right)=-2 K_{j, 1}\left(x_{j}, x_{j}\right) .
$$

Using the same approach used for Eqs. (18) and (19), we get the following formula for the potential $p_{j}\left(x_{j}\right)$ :

$$
p_{j}\left(x_{j}\right)=-2 \bar{K}_{j, 2}\left(x_{j}, x_{j}\right) .
$$

\section{Conclusions}

In this paper, we developed a procedure for finding the unknown potential in the Dirac equation on metric graphs using a given scattering matrix. In this case, the solutions for the inverse scattering problem were obtained by solving Gelfand-Levitan-Marchenko integral equations. The above results can be used in different practically important problems for emerging nanotechnologies. In addition, they can be useful for solving some nonlinear evolution equations on metric graphs such as nonlinear Schrodinger and KdV equations.

\section{References}

[1] Gnutzmann S. and Smilansky U. Quantum graphs: Applications to quantum chaos and universal spectral statistics. Advances in Physics, 55(5-6) July-October, P. 527-625 (2006).

[2] Kottos T., Smilansky U. Periodic Orbit Theory and Spectral Statistics for Quantum Graphs. Annals of Physics, 274, P. 76-124 (1999).

[3] Shapira T., Smilansky U. Quantum graphs which sound the same. Math., Phys., Chem., 213, P. 17-29 (2006).

[4] Kuchment P. Quantum graphs: I. Some basic structures. Waves Random Media, 14, P. S107-S128 (2004).

[5] Levitan B.M., Sargsyan I.S. Shturm-Liuville and Dirac operators. Moscow: Nauka, 432 p. (1988).

[6] Gasymov M.G., Levitan B.M. Definition of the Dirac system on scattering phase. Dokl. Akad. Nauk USSR, 167(6), P. 1219-1222 (1966).

[7] Asymova G.M., Guseynov I.M. Inverse problem of the scattering theory for grid equation of the First order. Dokl. Acad. Nauk Az SSR, 39(11), P. 12-15 (1983).

[8] Khanmamedov Ag. Kh. Inverse scattering problem for the difference Dirac operator on a half-line. Dokl. Akad. Hauk, 424(5), P. 597-598 (2009).

[9] Bolte J., Harrison J. Spectral Statistics for the Dirac Operator on Graphs. arXiv:nlin.CD/0210029, 1(15) Oct, P. 1-28 (2002).

[10] Gerasimenko N.I., Pavlov B.S. Scatterng problem on noncompact graphs. Theor. Mathem. Phys., 74(3), P. 345-359 (1988).

[11] Gerasimenko N.I. Inverse scattering problem on the noncompact graph. Theor. Mathem. Phys., 75(2), P. 187-200 (1988).

[12] Zakharov V.B. and Shabat A.B. Exact theory of two-dimensional self-focusing and one-dimensional self-modulation of waves in nonlinear media. Sov. Phys. JETP., 34(1), P. $62-69$ (1972). 